\title{
Protocol
}

\section{Rationale, study design and methodology of the hypertension registry: a Pan-India, prospective, longitudinal study to assess management and real-world outcomes of high-risk essential hypertension}

\author{
Jabir Abdullakutty', Jay Shah², Abraham Oomman³, J. Ezhilan', \\ N. C. Krishnamani ${ }^{5}$, Girish Navasundi ${ }^{6}$, Mohammed Y. Khan ${ }^{7 *}$, \\ Kumar Gaurav ${ }^{7}$, Sucheta Pandit ${ }^{7}$, Amey Mane ${ }^{7}$
}

\begin{abstract}
${ }^{1}$ Department of Cardiovascular Clinical Research, Lisie Hospital Rd, North Kaloor, Kaloor, Ernakulam, Kerala, India ${ }^{2}$ Department of Cardiology, HCG Hospital, Ellisbridge, Ahmedabad, Gujarat, India

${ }^{3}$ Department of Cardiology, Apollo Hospital, Chennai, Tamil Nadu, India

${ }^{4}$ Department of Peripheral and Vascular, The Madras Medical Mission Hospital, Chennai, Tamil Nadu, India

${ }^{5}$ Department of Non-invasive Cardiology, Fortis-Shalimarbagh, New Delhi, India

${ }^{6}$ Department of Cardiology, Apollo Hospital, Bannerghatta Road, Bangalore, Karnataka, India

${ }^{7}$ Department of Medical Affairs, Dr. Reddy's Laboratories Ltd, Ameerpet, Hyderabad, India
\end{abstract}

Received: 20 May 2021

Revised: 12 July 2021

Accepted: 14 July 2021

\author{
*Correspondence: \\ Mohammed Y. Khan, \\ E-mail: doctorkhan26@gmail.com
}

Copyright: () the author(s), publisher and licensee Medip Academy. This is an open-access article distributed under the terms of the Creative Commons Attribution Non-Commercial License, which permits unrestricted non-commercial use, distribution, and reproduction in any medium, provided the original work is properly cited.

\section{ABSTRACT}

Background: India contributes significantly towards a large part of the worldwide epidemic of hypertension (HTN) and its associated complications. As, there are limited longitudinal studies available in India to understand its occurrence over time, this Pan-India longitudinal study will aid to assess the real world outcomes of HTN across the country.

Methods: This was a prospective, multi-centered, longitudinal, observational study investigating a large COHORT of people with HTN across India over a period of one year. The primary objective of this study was to evaluate blood pressure (BP) control and clinical outcomes in high-risk hypertensive patients distributed over 5 visits (including baseline visit). The secondary objective is to assess the co-morbidities/risk factors in different clinical settings across India. Participants (4,000) with HTN will be included from 200 centres across India and data will be recorded for the use of anti-hypertensives, demographics, socio-economic status, anthropometric measurements, family history, personal history, risk factors, co-morbid conditions and physician treatment preferences. Overall, clinical practice patterns were assessed for their relationship with clinical outcomes.

Conclusions: This study is expected to reveal the trends in complications associated with HTN, treatment strategies used by physicians, and correlation among treatment, control and complications of HTN within the Indian context. The outcome of this study will help to identify the burden of HTN, along with pin-pointing the emergence of earlyonset complications and dose titration patterns. This will eventually help to develop person-centred care and facilitate public health agencies to invest appropriate resources in the management of high-risk HTN across India.

Trial registration: This is a real world evidence generation study which has been registered on Clinical Trials Registry-India (CTRI). The registration number for the same is CTRI/2020/07/026524.

Keywords: Hypertension, Coronary heart disease, Blood pressure, Study endpoints, Inclusion/exclusion criteria 


\section{INTRODUCTION}

HTN is an important public health problem in India and is ranked as the third most important risk factor for attributable burden of disease in South Asia. ${ }^{1}$ Various studies from India have reported its prevalence at $25 \%$ in urban and $10 \%$ in rural population.,3 Estimates by the WHO put HTN prevalence at $33.2 \%$ in men and $31.7 \%$ in women, with an overall prevalence of $32.5 \% .^{4,5}$ Furthermore, worldwide data in 2005 reported that the projected rates for HTN by 2025 will be $22.9 \%$ for men and $23.6 \%$ for women in India. ${ }^{6}$

In a recent report on HTN prevalence in India, substantial variations in HTN prevalence by age, rural versus urban location and state across India have been observed. Similarly, data from Maharashtra, report wide variations in HTN prevalence across different districts. ${ }^{8}$

Thus, gathering detailed information related to HTN variations could be further used to target and educate relevant programs across different states and regions in India.

\section{High-risk and very high-risk HTN}

The high-risk and the very high-risk categories are hypertensive patients which have co-existing cerebrovascular disease (CVD), coronary heart disease (CHD), peripheral vascular disease, myocardial infarction (MI), hyperlipidaemia, kidney disease, geriatric population and diabetics. ${ }^{9}$

\section{Guidelines on HTN}

Considering the European society of cardiology (ESC) and European society of HTN (ESH) guidelines for the management of arterial HTN, the drug treatment is recommended for high normal BP (130-139/85-89 $\mathrm{mmHg}$ ) individuals when cardiovascular risk is very high due to established CVD, especially CAD. ${ }^{10}$ The recommended target blood pressure by the Indian guidelines on HTN (IGH-IV) is lower in high-risk hypertensives than those without any associated risks (Table 1). ${ }^{11}$

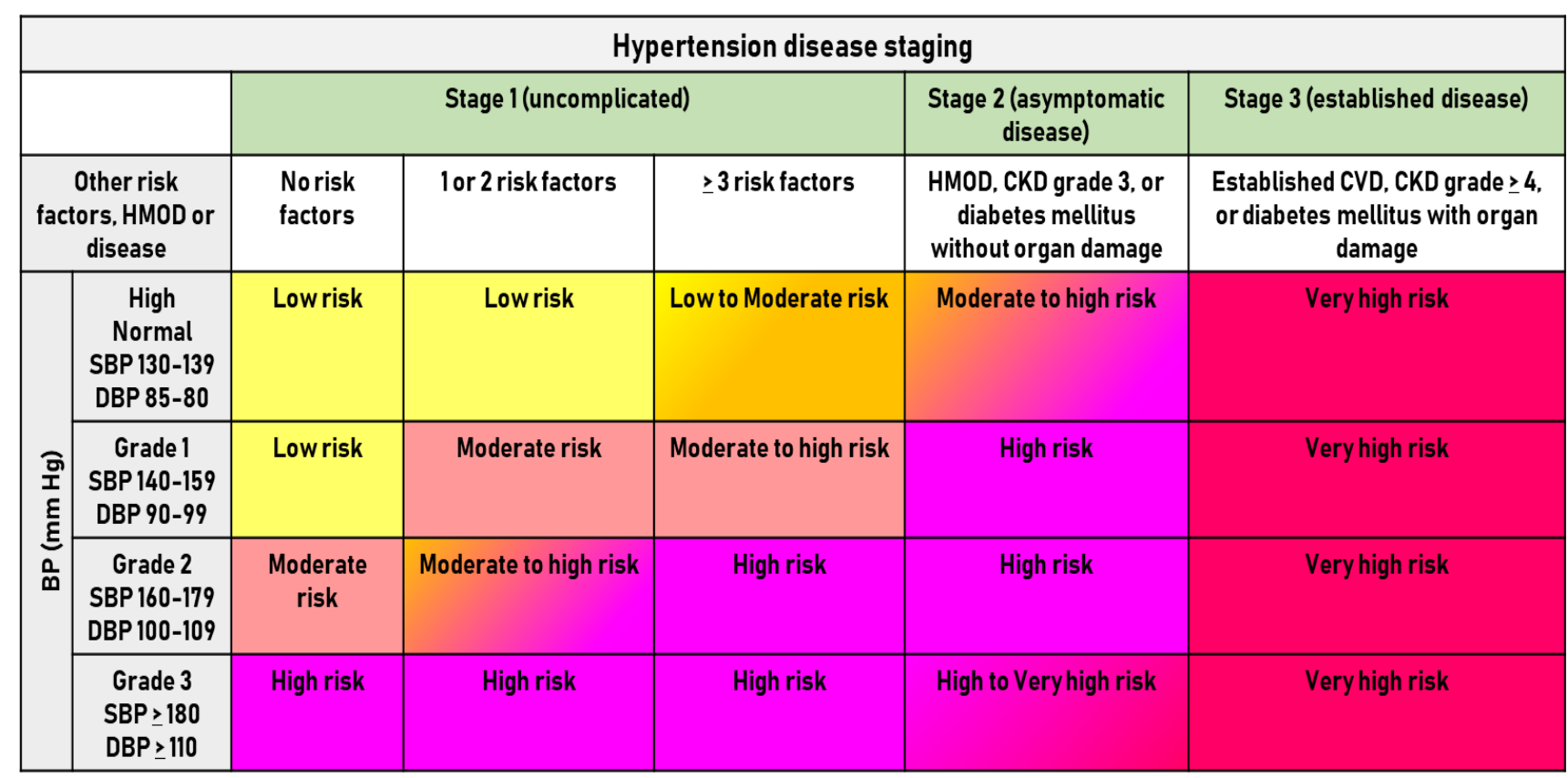

HMOD-hypertension-mediated organ damage; CKD-chronic kidney disease; CVD-cardiovascular disease; BP-blood pressure.

Figure 1: Risk factors for hypertension. ${ }^{9}$

Table 1: Threshold to start treatment and target BP as per IGH-IV.11

\begin{tabular}{|lll|}
\hline Subjects & $\begin{array}{l}\text { Threshold to start treatment } \\
(\geq)\end{array}$ & Target BP \\
\hline Age $<\mathbf{6 5}$ years & & $120-130 / 70-80$ \\
\hline High ASCVD risk, low ASCVD risk and Age 65-80 years & $140 / 90$ & $130-140 / 70-80$ \\
\hline Age $>80$ years & $140-150 / 90$ & \\
\hline With other risk factors & & $120-130 / 70-80$ \\
\hline CAD and heart failure & $130 / 80$ & $130-140 / 70-80$ \\
\hline Diabetes, history of stroke, TIA and CKD & $140 / 90$ & \\
\hline
\end{tabular}




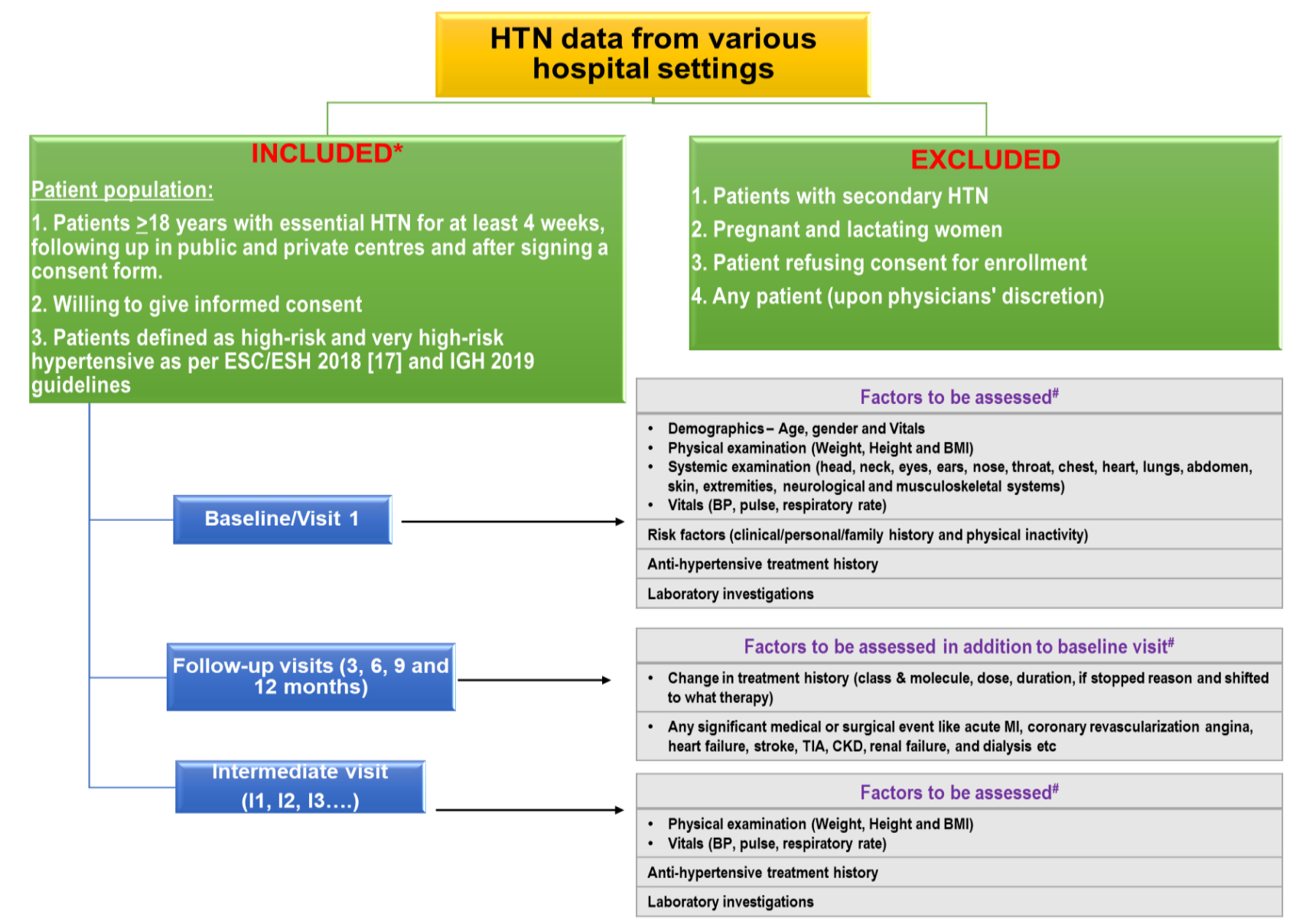

*refer study population section, \#refer Table 2 for detailed assessments.

Figure 2: Overall study design including inclusion and exclusion criterion.

\section{Study rationale}

Despite the high prevalence of HTN in India, the awareness, treatment and control status are low in urban as well as rural populations in India. ${ }^{12}$ This could be attributed to a variety of socio-economic factors including low educational status, poverty, rural residence, as well as physiological factors like obesity. ${ }^{13}$

As high-risk hypertensives are more prone to fatal and non-fatal outcomes early in life, the recommended BP target in these individuals is much tighter. ${ }^{14}$ Therefore, comprehensive HTN management should not only focus on reducing $\mathrm{BP}$, but also lowering the $\mathrm{CV}$ risk by lifestyle measures, lipid management, smoking cessation and regular exercise. ${ }^{15}$ Further, it is a very wellestablished fact that control of BP can decrease the CV risk and the latest ESH/ESC guidelines recommend appropriate lifestyle measures and anti-hypertensive therapy to this. ${ }^{10}$

Several safe and effective medications are available for the treatment of HTN which include drugs such as diuretics, beta-blocking agents, calcium channel blockers $(\mathrm{CCBs})$, angiotensin converting enzyme (ACE) inhibitors and angiotensin receptor blockers (ARB). However, due to the availability of multiple drugs and varying level of awareness about the national and international guidelines amongst practitioners in India, the achievement of BP target becomes challenging. Therefore, it becomes necessary to collect information regarding the clinical presentation of HTN in Indians and the common trends and practices followed by doctors for its treatment. This data would provide an insight into the current practices and its success in BP goal achievements especially in high-risk individuals.

This study aims to collect real-world data from the Indian population about BP control with the use of antihypertensive drugs used in high-risk hypertensives. Additionally, we would also collect data of patient demographics, socio-economic status, anthropometric measurements, family history, personal history, risk factors, co-morbid conditions, physician treatment preferences and clinical BP data.

This information would help us assess the BP control parameters and treatment compliance in high-risk hypertensives. Further, data obtained in this study would help us to understand the underlying reasons for a lack of 
BP control in high-risk hypertensives and will aid in formulating recommendations and treatment protocols for this subset of hypertensive patients.

\section{METHODS}

\section{Study design}

\section{Description of study design}

This was a prospective, multicentric, observational, realworld study to determine multiple aetiologies, varying presentation, clinical practice patterns, drug utilization patterns and outcomes of HTN in high-risk hypertensive patients in India.

Upto 4,000 patients for the study was included from all types of hospital settings (private/public, primary healthcare/clinics/secondary healthcare/tertiary healthcare, rural/urban). The data was collected from about 200 study sites/centres across India as highlighted in Figure 2.

The study evaluations were performed at baseline and at every 3 months interval with a window period of \pm 7 days. Any visit other than above mentioned visits was documented as intermediate visit.

Detailed assessments for visits are provided in Table 2 (time and events schedule). BP will be measured with a standard sphygmomanometer in the same arm throughout the study.

Sites enrolling patients in this study recorded data on electronic case report forms (eCRFs) using electronic data capture (EDC) system. A designated clinical research organization (CRO) will capture, check, store and analyze the data.

\section{Study endpoints}

\section{Primary endpoints}

Proportion of patients achieving their BP goals as per ESC/ESH 2018 and IGH 2019 guidelines for the management of arterial HTN at 12 months. ${ }^{10,16}$

\section{Secondary endpoints}

Proportion of patients with macro-vascular clinical outcomes: all-cause mortality, MI, stroke, CAD, HF and peripheral arterial disease (PAD) at 12 months.

Proportion of patients with micro-vascular clinical outcomes: retinopathy, nephropathy and neuropathy at 12 months.

Proportion of high-risk hypertensive patients with both modifiable and non-modifiable risk factors at baseline.
Proportion of patients with co-morbidities at baseline defined by diagnosis (DM, dyslipidaemias, CKD, CVD, HF/stroke/TIA, MI, neuropathy, retinopathy and/or other).

Clinical practice patterns (diagnosis parameters considered to make diagnosis, routine diagnostic measures), treatment and drug utilization patterns, investigations (investigations performed to evaluate the patients) and relationship to clinical outcomes.

\section{Exploratory endpoints}

Resting heart rate will be evaluated from baseline to end of study.

\section{Clinical outcomes}

The outcomes of interest are micro-vascular outcomes comprising retinopathy, nephropathy, neuropathy and macro-vascular outcomes which include all-cause mortality, MI, stroke, CAD, HF, PAD.

For definitions of micro-vascular and macro-vascular outcomes refer to Appendix 1.

\section{Adherence to medication}

Patient compliance to medication prescribed would be assessed based on a questionnaire at each visit. Kindly refer Appendix 1 for the questionnaire.

\section{Study population}

\section{Inclusion criteria}

Patient willing to give informed consent, patients (both males and females) 18 years or older with essential HTN (as per ESC/ESH 2018 and IGH 2019 guidelines) for at least 4 weeks, following up in public and private centres and after signing a consent form were included in the study. Patients defined as high-risk and very high-risk hypertensive as per ESC/ESH 2018 and IGH 2019 guidelines as per the checklist below: ${ }^{10,17}$

Grade 3 HTN (SBP $\geq 180 \mathrm{mmHg}$ or DBP $\geq 110 \mathrm{mmHg}$ ) with any one of the following: no risk, 1-2 risk factor or $\geq 3$ CV risk factors; asymptomatic HMOD (HTNmediated organ damage); CKD grade 3 (eGFR $>30$ to 59 $\left.\mathrm{ml} / \mathrm{min} / 1.73 \mathrm{~m}^{2}\right)$, or grade $4\left(\mathrm{eGFR}<30 \mathrm{ml} / \mathrm{min} / 1.73 \mathrm{~m}^{2}\right)$; DM without/with organ damage; established CVD.

Grade 2 hypertension (SBP 160-179 mmHg OR DBP 100-109 $\mathrm{mmHg}$ ) with any one of the following: uncomplicated HTN with $\geq 3 \quad \mathrm{CV}$ risk factors; asymptomatic HMOD (hypertension-mediated organ damage); CKD grade 3 (eGFR > 30 to $59 \mathrm{ml} / \mathrm{min} / 1.73 \mathrm{~m}^{2}$ ) or grade $4\left(\mathrm{eGFR}<30 \mathrm{ml} / \mathrm{min} / 1.73 \mathrm{~m}^{2}\right)$; DM without/with organ damage; established CVD. 
Grade 1 HTN (SBP 140-159 mmHg OR DBP 90-99 $\mathrm{mmHg}$ ) with any one of the following: asymptomatic HMOD (hypertension-mediated organ damage); CKD grade $3\left(\mathrm{eGFR}>30\right.$ to $\left.59 \mathrm{ml} / \mathrm{min} / 1.73 \mathrm{~m}^{2}\right)$ or grade 4 (eGFR $\left.<30 \mathrm{ml} / \mathrm{min} / 1.73 \mathrm{~m}^{2}\right)$; DM without/with organ damage; established CVD.

\section{Exclusion criteria}

Patients with secondary HTN (secondary HTN is caused by an identifiable underlying primary cause), pregnant and lactating women, patients refusing consent for enrolment and any patient as per the discretion of the physician were excluded from the study.

Table 2: Time and schedule of events.

\begin{tabular}{|c|c|c|c|c|c|c|c|}
\hline \multirow{2}{*}{\multicolumn{2}{|c|}{ Visit $\rightarrow$}} & \multirow{2}{*}{$\begin{array}{l}\text { Screening phase and } \\
\text { enrolment phase } \\
1\end{array}$} & \multicolumn{5}{|c|}{ Observational and follow up phase } \\
\hline & & & 2 & 3 & 4 & 5 & \multirow[b]{2}{*}{ Intermediate } \\
\hline $\begin{array}{l}\text { S. } \\
\text { No. }\end{array}$ & $\begin{array}{l}\text { Month } \rightarrow \\
\text { Activity } \downarrow\end{array}$ & -14 to 0 & $\begin{array}{l}\text { Month } \\
3 \pm 7 \\
\text { days }\end{array}$ & $\begin{array}{l}\text { Month } \\
6 \pm 7 \\
\text { days }\end{array}$ & $\begin{array}{l}\text { Month } \\
9 \pm 7 \\
\text { days }\end{array}$ & $\begin{array}{l}\text { Month } \\
12 \pm 7 \\
\text { days/end } \\
\text { of study }\end{array}$ & \\
\hline 1. & Informed Consent & $\mathrm{X}$ & & & & & \\
\hline 2. & $\begin{array}{l}\text { Medical history and } \\
\text { demographics }\end{array}$ & $\mathrm{X}$ & & & & & \\
\hline 3. & Inclusion/exclusion criteria & $\mathrm{X}$ & & & & & \\
\hline 4. & Physical examination & $\mathrm{X}$ & $\mathrm{X}$ & $\mathrm{X}$ & $\mathrm{X}$ & $\mathrm{X}$ & $\mathrm{X}$ \\
\hline 5. & Vital signs & $\mathrm{X}$ & $\mathrm{X}$ & $\mathrm{X}$ & $\mathrm{X}$ & $\mathrm{X}$ & $\mathrm{X}$ \\
\hline 6. & Clinical outcomes & & $\mathrm{X}$ & $\mathrm{X}$ & $\mathrm{X}$ & $\mathrm{X}$ & $\mathrm{X}$ \\
\hline 7. & $\begin{array}{l}\text { Anti-hypertensive treatment } \\
\text { history }\end{array}$ & $\mathrm{X}$ & $\mathrm{X}$ & $\mathrm{X}$ & $\mathrm{X}$ & $\mathrm{X}$ & $\mathrm{X}$ \\
\hline 8. & $\begin{array}{l}\text { Management of clinical } \\
\text { outcome }\end{array}$ & & $\mathrm{X}$ & $\mathrm{X}$ & $\mathrm{X}$ & $\mathrm{X}$ & $\mathrm{X}$ \\
\hline \multicolumn{8}{|c|}{ Laboratory and other investigations record ${ }^{v}$} \\
\hline 9. & ECG, ECHO & $\mathrm{X}$ & $\mathrm{X}$ & $\mathrm{X}$ & $\mathrm{X}$ & $\mathrm{X}$ & $\mathrm{X}$ \\
\hline 10. & Liver function tests & $\mathrm{X}$ & $\mathrm{X}$ & $\mathrm{X}$ & $\mathrm{X}$ & $\mathrm{X}$ & $\mathrm{X}$ \\
\hline 11. & Lipid profile & $\mathrm{X}$ & $\mathrm{X}$ & $\mathrm{X}$ & $\mathrm{X}$ & $\mathrm{X}$ & $\mathrm{X}$ \\
\hline 12 & Renal function tests & $\mathrm{X}$ & $\mathrm{X}$ & $\mathrm{X}$ & $\mathrm{X}$ & $\mathrm{X}$ & $\mathrm{X}$ \\
\hline & $\begin{array}{l}\text { Fasting plasma glucose/random } \\
\text { plasma glucose }\end{array}$ & $\mathrm{X}$ & $\mathrm{X}$ & $\mathrm{X}$ & $\mathrm{X}$ & $\mathrm{X}$ & $\mathrm{X}$ \\
\hline 14. & $\mathrm{HbA1c}$ & $\mathrm{X}$ & $\mathrm{X}$ & $\mathrm{X}$ & $\mathrm{X}$ & $\mathrm{X}$ & $\mathrm{X}$ \\
\hline 15. & $\begin{array}{l}\text { Serum electrolytes }(\mathrm{Na}, \mathrm{K}, \mathrm{Cl} \text {, } \\
\mathrm{Ca})\end{array}$ & $\mathrm{X}$ & $\mathrm{X}$ & $\mathrm{X}$ & $\mathrm{X}$ & $\mathrm{X}$ & $\mathrm{X}$ \\
\hline 16. & Haematology & $\mathrm{X}$ & $\mathrm{X}$ & $\mathrm{X}$ & $\mathrm{X}$ & $\mathrm{X}$ & $\mathrm{X}$ \\
\hline & Urine microscopy & $\mathrm{X}$ & $\mathrm{X}$ & $\mathrm{X}$ & $\mathrm{X}$ & $\mathrm{X}$ & $\mathrm{X}$ \\
\hline & Urine chemical examinations & $\mathrm{X}$ & $\mathrm{X}$ & $\mathrm{X}$ & $\mathrm{X}$ & $\mathrm{X}$ & $\mathrm{X}$ \\
\hline & Cardiac doppler & $\mathrm{X}$ & $\mathrm{X}$ & $\mathrm{X}$ & $\mathrm{X}$ & $\mathrm{X}$ & $\mathrm{X}$ \\
\hline & Cardiac angiography & $\mathrm{X}$ & $\mathrm{X}$ & $\mathrm{X}$ & $\mathrm{X}$ & $\mathrm{X}$ & $\mathrm{X}$ \\
\hline & Fundus examination & $\mathrm{X}$ & $\mathrm{X}$ & $\mathrm{X}$ & $\mathrm{X}$ & $\mathrm{X}$ & $\mathrm{X}$ \\
\hline & Any other & $\mathrm{X}$ & $\mathrm{X}$ & $\mathrm{X}$ & $\mathrm{X}$ & $\mathrm{X}$ & $\mathrm{X}$ \\
\hline
\end{tabular}

\section{Study procedures}

The patients were included from all types of hospital settings (private/public, primary healthcare/clinics/ secondary healthcare/tertiary healthcare, rural/urban) for the study. Following information about the evaluations were captured for the study purpose.

\section{Clinical evaluations}

During patient visits following clinical evaluations were done as a part of routine clinical assessment like clinical, personal, family history; vitals recorded, physical and systemic examination; clinical outcomes, management of clinical outcomes; anti-hypertensive treatment history

The schedule of assessments is given in Table 2 .

\section{Record of blood pressure measurements}

Data of BP measurements during the patient's clinic/hospital visits using a standard sphygmomanometer (mercury/digital/aneroid) was captured in the registry. An 
attempt would be made to capture maximum BP recordings.

Data of any other BP recordings done during the study period like ambulatory BP monitoring (ABPM) or homebased BP monitoring (HBBM) was captured. Data for such patients with ABPM, HBBM and ECG was analysed as separate sub-groups.

\section{Record of laboratory and other investigations}

All laboratory and other investigations were performed at the discretion of the investigator at baseline and every visit based on his/her clinical judgment and in the interest of patient safety. However, no investigations were performed for the study purpose.

\section{Study schedule}

\section{Screening}

The patients for the study were included from all types of hospital settings (private/public, primary healthcare/clinics/secondary healthcare/tertiary healthcare, rural/urban). Patients were screened based on the inclusion and exclusion criteria of the study.

\section{Enrolment/baseline visit}

Each patient was enrolled into the study after obtaining informed consent from the patient or his/her legally authorized representative/impartial witness. Patient eligibility for participation in the study were assessed. Once patient met all eligibility criteria, he/she was enrolled in the study.

Refer Table 2 for scheduled baseline activities.

\section{Follow up visits}

After enrolment, the follow-up visits were at 3 months intervals, at 3, 6, 9 and 12 months. However, no visits were mandatory and reasons for each follow-up was captured in the eCRF.

Refer Table 2 for scheduled activities at each visit.

Final study visit

The final study visit was at the end of 12 months after enrolment/baseline. Refer Table 2 for scheduled activities. For intermediate visit, the data was recorded as per scheduled follow up visits.

\section{Study population}

Data from patients (irrespective of 12 months completion of study period) collected during the study period was used for study assessments.

\section{Sample size}

It was planned to include data of upto 4000 high-risk hypertensive patients for the study from upto 200 study sites/centres across India. This was a single arm noncomparative observational study aiming for neither noninferiority nor superiority to another comparator or placebo. Therefore, sample size did not need to fulfil statistical based value and there was no hypothesis to test or compare statistically.

\section{Description of the analyses}

The data was presented using descriptive statistical methods.

For continuous variables number of non-missing observations (n), mean, standard deviation (SD), median, maximum and minimum (range) will be presented.

For categorical data, number of patients (n) out of total exposed patients along with percentage will be calculated for each category of the categorical variable.

Along with the overall results, results would be stratified based on gender, co-morbidities, micro-vascular complications and macro-vascular complications. Data of patients with ABPM and HBBM will be evaluated separately as sub-groups.

All statistical analyses will be performed using the SAS System software.

\section{Participant enrolment and follow up}

It was planned to include data of up to 4,000 high-risk HTN patients for this study. These patients were followed up for 12 months and data on the clinical outcomes over 12 months were captured and evaluated using descriptive statistics.

\section{Ethics}

\section{Institutional review board/ethics committee}

Before initiating the study, the investigator/institution obtained approval/favourable opinion from the IRB/IEC for the trial protocol, informed consent form and any other written information that were provided to patients/subjects.

\section{Participant confidentiality}

Prior to the patient's enrolment in the study, the patient's consent was required to gain direct access to their medical records for data verification purposes. All personal data of the patients were treated as confidential. 


\section{Current status}

As of 2021, the study has started recruitment by enrolling participants.

\section{DISCUSSION}

The increasing trend in the prevalence of HTN and its associated complications contributing significantly to overall morbidity and mortality has been a constant challenge in India. ${ }^{2,3,6}$ Most of the studies that have been performed to date in India are population-based crosssectional studies and in this regard, HTN registry is a first-of-its-kind prospective, long-term, longitudinal study conducted in a large cohort of people with essential HTN. ${ }^{2,3,5}$ This study aimed not only to precisely determine its disease burden in India, but also to evaluate the development of macro-vascular and micro-vascular complications in these individuals over time and explore their regional occurrence. In this study, the role of effective treatment strategy in preventing HTN complications and the course of the disease will be assessed. In previous population-based studies in India, the prevalence of HTN and the associated macro-vascular complications were investigated at a much smaller scale., $3,5,12$ The Pan-India, urban and peri-urban representation of participants in this study can provide valuable insights into the relationship between HTN related complications and BP control over time at both urban and rural levels. This study was expected to help in understanding the emergence of complications at a relatively early age, thereby preventing the comorbidities that could emerge later. As compared to its western counterparts, it was well established that the diagnosis of HTN in India takes place quite late in the disease cycle and that the associated complications appear a decade earlier. ${ }^{4}$ Furthermore, literature from population-based studies in India indicate that the disease diagnosis remains undetected in about half of the population and this further supports the observation of an outsized majority of individuals having complications at the time of diagnosis itself., ${ }^{7,8}$ Overall, we expect that the rate of complications of HTN will be truly representative of the real-world situation in India and this study will capture critical information on following common trends, increased use of combinations, dose titration patterns, patient and physician behaviours. Our study may help in identifying the role of an optimal therapy in preventing HTN complications and reducing its overall management cost. Through this study, a person's journey, his/her response to varied treatments over a period and therefore the possible unmet needs can be recognized. Additionally, the study may provide an insight into changes in physician preferences and treatment patterns with the introduction of new therapies.

Based on other international registries, this will be the registry of unselected HTN patients in India with a 1 year follow up period and the major limitation of this longitudinal study was that some patients may have an incomplete and an interrupted follow up. We hoped that after this project is successfully completed, we will be able to expand this initiative with larger studies and longer follow-up periods. We expect that this registry will generate data that will help in the planning and execution of well-powered studies in future.

The strengths of this study included a Pan-India representation of investigators and a large, enrolled COHORT that will provide an in-depth understanding of the disease landscape and real-world management of essential HTN in India. In conclusion, the study design will reflect real-life normal routine practice of management of essential HTN, and the outcomes are expected to be a true reflection of the real world.

\section{CONCLUSION}

This study intends to assess the trends of HTN, its associated complications and treatment strategies within the Indian context. With the collective understanding of all the objectives of this study, public health agencies can be propelled to invest in relevant resources for its management. Through this approach, person centred care can be developed and both treatment targets and treatment strategies can be tailored. The study can therefore be an impetus for redefining the approach towards management and control of HTN in India.

\section{ACKNOWLEDGEMENTS}

The authors thank Pharmasolutions360 for providing medical writing assistance.

Funding: Funded by Dr. Reddys Laboratories, India Conflict of interest: MYK, SP, AM and KG are employees of Dr. Reddy's Laboratories and may own stock. JA, JS, $A O, J E, N C K$ and GNB are members of the advisory board for Dr. Reddy's Laboratories

Ethical approval: The study was approved by the Institutional Ethics Committee

\section{REFERENCES}

1. Lim SS, Vos T, Flaxman AD, Danaei G, Shibuya K, Adair-Rohani $\mathrm{H}$, et al. A comparative risk assessment of burden of disease and injury attributable to 67 risk factors and risk factor clusters in 21 regions, 1990-2010: a systematic analysis for the Global Burden of Disease Study 2010. Lancet. 2012;380(9859):2224-60.

2. Thankappan KR, Sivasankaran S, Sarma PS, Mini G, Khader SA, Padmanabhan P, et al. Prevalencecorrelates-awareness-treatment and control of hypertension in kumarakom, kerala: baseline results of a community-based intervention program. Indian Heart J. 2006;58(1):28-33.

3. Das SK, Sanyal K, Basu A. Study of urban community survey in India: growing trend of high 
prevalence of hypertension in a developing country. Int J Med Sci. 2005;2(2):70-8.

4. WHO. Fact sheet: Noncommunicable diseases country profiles, 2011. Available at: https://www.who.int/nmh/publications/ncd_profiles 2011/en/. Accessed on 5 May 2021.

5. Hypertension Study Group. Prevalence, awareness, treatment and control of hypertension among the elderly in Bangladesh and India: a multicentre study. Bull World Health Organ. 2001;79(6):490500 .

6. Kearney PM, Whelton M, Reynolds K, Muntner P, Whelton PK, He J. Global burden of hypertension: analysis of worldwide data. Lancet (London, England). 2005;365(9455):217-23.

7. Geldsetzer P, Manne-Goehler J, Theilmann M, Davies JI, Awasthi A, Vollmer S, et al. Diabetes and Hypertension in India: A Nationally Representative Study of 1.3 Million Adults. JAMA Intern Med. 2018;178(3):363-72.

8. Bhise MD, Patra S. Prevalence and correlates of hypertension in Maharashtra, India: a multilevel analysis. PloS One. 2018;13(12):0191948.

9. Williams B, Mancia G, Spiering W, Rosei EA, Azizi M, Burnier M, et al. 2018 practice guidelines for the management of arterial hypertension of the European society of hypertension and the European society of cardiology: ESH/ESC task force for the management of arterial hypertension. J Hypertens. 2018;36(12):2284-309.

10. Cuspidi C, Tadic M, Grassi G, Mancia G. Treatment of hypertension: The ESH/ESC guidelines recommendations. Pharmacol Res. 2018;128:31521.

11. Shah SN, Munjal YP, Kamath SA, Wander GS, Mehta N, Mukherjee S, et al. Indian guidelines on hypertension-IV (2019). J Hum Hypertens. 2020;34(11):745-58.

12. Anchala R, Kannuri NK, Pant H, Khan H, Franco $\mathrm{OH}$, Angelantonio ED, et al. Hypertension in India: a systematic review and meta-analysis of prevalence, awareness, and control of hypertension. J Hypertens. 2014;32(6):1170-7.

13. WHO. Fact sheet: BMI Classification. Global Database on Body Mass Index, 2006. Available at: https://web.archive.org/web/20090418042851/http:// www.who.int/bmi/index.jsp?introPage=intro_3.htm. Accessed on 5 May 2021.

14. Calhoun DA, Jones D, Textor S, Goff DC, Murphy TP, Toto RD, et al. Resistant hypertension: diagnosis, evaluation, and treatment. A scientific statement from the American heart association professional education committee of the council for high blood pressure research. Hypertension. 2008;51:1403-19.
15. Gupta R, Guptha S. Strategies for initial management of hypertension. Indian $\mathrm{J}$ Med Res. 2010;132(5):531-42.

16. Association of Physicians of India. Indian guidelines on hypertension (I.G.H.)-III. 2013. J Assoc Physicians India. 2013;61(2):6-36.

17. Williams B, Mancia G, Spiering W, Rosei EA, Azizi M, Burnier M, et al. 2018 ESC/ESH Guidelines for the management of arterial hypertension: the task force for the management of arterial hypertension of the European society of cardiology and the European society of hypertension: the task force for the management of arterial hypertension of the European society of cardiology and the European society of hypertension. J Hypertens. 2018;36(10):1953-2041.

18. Chatterjee S, Chattopadhyay S, Hope-Ross M, Lip PL. Hypertension and the eye: changing perspectives. J Hum Hypertens. 2002;16(10):66775.

19. Hart PD, Bakris GL. Hypertensive nephropathy: prevention and treatment recommendations. Expert Opin Pharmacother. 2010;11(16):2675-86.

20. Edwards L, Ring C, McIntyre D, Winer JB, Martin U. Cutaneous sensibility and peripheral nerve function in patients with unmedicated essential hypertension. Psychophysiology. 2008;45(1):141-7.

21. Thygesen K, Alpert JS, Jaffe AS, Chaitman BR, Bax JJ, Morrow DA, et al. Executive group on behalf of the joint European Society of cardiology (ESC)/American college of cardiology (ACC)/American heart association (AHA)/world heart federation (WHF) task force for the universal definition of myocardial infarction. Fourth universal definition of myocardial infarction. $\mathrm{J}$ Am Coll Cardiol. 2018;72:2231-64.

22. Kumar A, Cannon CP. Acute coronary syndromes: diagnosis and management, part I. Mayo Clin Proc. 2009;84(10):917-38.

23. Musuka TD, Wilton SB, Traboulsi M, Hill MD. Diagnosis and management of acute ischemic stroke: speed is critical. CMAJ. 2015;187(12):88793.

24. Ponikowski P, Voors AA, Anker SD, Bueno H, Cleland JG, Coats AJ, et al. 2016 ESC guidelines for the diagnosis and treatment of acute and chronic heart failure: the task force for the diagnosis and treatment of acute and chronic heart failure of the European society of cardiology (ESC). Developed with the special contribution of the heart failure association (HFA) of the ESC. Eur J Heart Fail. 2016;37(27):2129-200.

25. Olin JW, Sealove BA. Peripheral artery disease: current insight into the disease and its diagnosis and management. Mayo Clin Proc. 2010;85(7):678-92.

Cite this article as: Abdullakutty J, Shah J, Oomman A, Ezhilan J, Krishnamani NC, Navasundi BG, et al. Rationale, study design and methodology of the hypertension registry: a Pan-India, prospective, longitudinal study to assess management and real-world outcomes of high-risk essential hypertension. Int J Clin Trials 2021;8(3):223-32. 


\section{APPENDIX 1}

\section{Micro-vascular complications}

Hypertensive retinopathy: Degenerative changes to the retina due to uncontrolled hypertension and characterized by background retinopathy and retinal vascular changes (proliferative or non-proliferative) on fundoscopy examination. ${ }^{18}$

Hypertensive nephropathy: Declining eGFR ( $<90 \mathrm{ml} / \mathrm{min}$.), macroalbuminuria (a random urine albumin/creatinine ratio $>300 \mathrm{mg} / \mathrm{g}$ ), microalbuminuria (a random urinealbumin/creatinine ratio 30-300 mg/g) or presence of CKD. ${ }^{19}$

Hypertensive neuropathy: Presence of symmetrical, bilateral distal motor and sensory impairment with a graded increase in severity distally. ${ }^{20}$

\section{Macro-vascular complications}

All-cause mortality: Death due to any cause.

MI: Symptoms of MI (chest pain, dyspnea, nausea, unexplained weakness or a combination of these symptoms) and ECG changes of MI (ST elevation or depression, $\mathrm{T}$ wave inversion or hyper-acute $\mathrm{T}$ waves) or elevated cardiac troponin $\mathrm{T}$ and troponin I levels. MI in patients with chest discomfort or other ischaemic symptoms, who develop new ST segment elevations in two contiguous leads or new bundle branch blocks with ischaemic repolarization patterns as an ST-elevation MI (STEMI). In contrast, patients without ST-segment elevation at presentation are usually designated non-ST-elevation MI (NSTEMI). A diagnosis of NSTEMI can be achieved when the ischemia is sufficiently severe to cause myocardial damage that can result in the release of a biomarker of myocardial necrosis into the circulation (cardiac-specific troponins $\mathrm{T}$ or I or muscle and brain fraction of creatine kinase CK-MB). ${ }^{21}$

CHD/CAD: CHD/CAD, also called IHD and/or atherosclerotic heart disease is a pathological process characterized by atherosclerotic plaque accumulation in the epicardial arteries, whether obstructive or non-obstructive. The nature of the CAD process results in multifarious clinical presentations, which can be categorized as either acute coronary syndromes (ACS) or chronic coronary syndromes (CCS). The term ACS refers to any group of clinical symptoms compatible with acute myocardial ischemia and includes unstable angina (UA), NSTEMI and STEMI. Unstable angina exhibits 1 or more of 3 principal presentations: (1) rest angina (usually lasting > 20 minutes), (2) new-onset ( $<2$ months previously) severe angina and (3) a crescendo pattern of occurrence (increasing in intensity, duration, frequency or any combination of these factors). ${ }^{22}$

Stroke or CVD: Acute cerebrovascular insufficiency and cerebral infarction due to thrombotic occlusion of pre-cerebral artery or acute cerebrovascular accident. It also includes transient ischemic attack (TIA) which is a temporary period of cerebrovascular insufficiency and may not cause permanent damage. Arterial occlusion in ischemic stroke is most commonly embolic: either cardioembolic, from causes such as atrial fibrillation or valvular heart disease or arteroembolic, from atherosclerotic disease in the extracranial cervical carotid or vertebral artery. Haemorrhagic strokes are divided equally into intracerebral haemorrhage and subarachnoid haemorrhage. ${ }^{23}$

HF: HF is a clinical syndrome characterized by typical symptoms (e.g. breathlessness, ankle swelling and fatigue) that may be accompanied by signs (e.g. elevated jugular venous pressure, pulmonary crackles and peripheral oedema) caused by a structural and/or functional cardiac abnormality, resulting in a reduced cardiac output and/or elevated intra-cardiac pressures at rest or during stress. HF comprises a wide range of patients, from those with normal left ventricular ejection fraction (LVEF) (typically considered as $\geq 50 \%$; HF with preserved ejection fraction HFpEF) to those with reduced LVEF (typically considered as $<40 \%$; HF with reduced EF HFrEF). ${ }^{24}$

PAD: PAD, which comprises atherosclerosis of the abdominal aorta, iliac and lower-extremity arteries. Patients with PAD may experience a multitude of problems such as claudication, ischemic rest pain, ischemic ulcerations, gangrene or no symptoms at all. The ankle brachial index is an excellent screening test for the presence of PAD. A value below 0.9 is considered diagnostic of PAD. Imaging studies (duplex ultrasonography, computed tomographic angiography, magnetic resonance angiography, catheter-based angiography) may provide additional anatomic information. ${ }^{25}$ 


\section{Adherence to medication}

Data of patient compliance to medication prescribed would be assessed based on the below questionnaire at each visit:

1. Did you ever forget to take the medication: Yes/No

2. How often have you missed out on taking a dose in last one week? 0,1-2,3-6,7-10,>10.

3. How often have you missed the dose since the last visit to the doctor: Number of days

4. (i) Do you encounter any problems when you take medication? Yes/No;

If yes, what kind of problems do you experience? Mention the problem.

(ii) What was the most common reason for non-compliance? Treatment cost, no. of medication, frequency of medication, adverse effects, any other. 